Ref. No. JP0703354

Title: Electrogenerated Chemiluminescence of $\mathrm{ZnS}$ Nanoparticles in Alkaline Aqueous Solution

Response to referee comments

Our paper referred to above has been revised according to reviewer's comments.

The language mistakes were corrected.

\title{
Reviewer \#65
}

\section{Major revisions}

1. The band gap of the NPs is given on p. 4 as $4.1 \mathrm{eV}$, as expected larger than that of bulk ZnS. However, the emission is at about $2.8 \mathrm{eV}(\sim 440 \mathrm{~nm})$, which is close to that observed with bulk ZnS [e.g. in F.-R. F. Fan, P. Leempoel, and A. J. Bard, "Semiconductor Electrodes. 51. Efficient Electroluminescence at ZnS Electrodes in Aqueous Electrolytes," J. Electrochem. Soc., 130, 1866 (1983).] Bulk ZnS is often doped, e.g. with Al or Cu, and this can account for the emission. Is there a possibility that there is a small amount of doping of the ZnS NPs (it wouldn't take many atoms)? At least, this point should be considered.

Reviewer \#65 indicated that the ECL emission of ZnS NPs about $2.8 \mathrm{eV}(\sim 440$ $\mathrm{nm}$ ) is close to that observed with bulk $\mathrm{ZnS}$ and a possibility that there is a small amount of doping of the ZnS NPs. In order to explanation of this small band gap, the energy dispersive X-ray (EDX) measurements of $\mathrm{ZnS}$ NPs and $\mathrm{ZnS} / \mathrm{Zn}(\mathrm{OH})_{2}$ were conducted. The corresponding EDX analysis and explanation, Figure 5 were added in the revised text. 
To obtain a reasonable explanation for this small band gap, the energy dispersive X-ray (EDX) measurements of $\mathrm{ZnS} \mathrm{NPs}$ and $\mathrm{ZnS} / \mathrm{Zn}(\mathrm{OH})_{2}$ were conducted to qualitatively identify the chemical composition of the ZnS NPs. Figure 5 (a) clearly shows that $\mathrm{Zn}$ and $\mathrm{S}$ peaks are at their normal energies, which indicate that the sample is $\mathrm{ZnS}$. And no aluminum signal or copper signal was observed. Therefore, the ECL emission of the $\mathrm{ZnS}$ NPs could not be ascribed to the small amount of doping aluminum or copper in the $\mathrm{ZnS}$ NPs. This suggests that the ECL emission mechanism of $\mathrm{ZnS}$ NPs is different from that of the bulk $\mathrm{ZnS}$ electrode in aqueous electrolytes. From Figure 5 (b), we also clearly see that that the signals of $\mathrm{Zn}, \mathrm{S}, \mathrm{O}$, and $\mathrm{C}$. The carbon signal can be ascribed to the supporting material. The oxygen signal strongly suggests that the core/shell structure of $\mathrm{ZnS} / \mathrm{Zn}(\mathrm{OH})_{2} \mathrm{NPs}$ is formed.

2. The reviewer pointed out that the peroxydisulfate protocol used with $\mathrm{ZnS}$ NPs was similar to that with a bulk ZnS electrode in aqueous solution, which was reported in 1983.

The reference mentioned (Fan, F. R. F.; Leempoel, P.; Bard, A. J. J. Electrochem. Soc. 1983, 130, 1866.) was cited in our paper.

3. It would be useful to have some indication of the current levels used.

The relationship of the ECL intensity and the current is very complex in our system. In order to show the recorded current, Figure S4 ECL-potential curve and cyclic voltammograms in $0.10 \mathrm{M}$ NaOH contaning $1.0 \mathrm{mM} \mathrm{ZnS}$ NPs and $0.010 \mathrm{M} \mathrm{K} \mathrm{S}_{2} \mathrm{O}_{8}$ was added in the Supporting Information. We can see that the current levels used was about $\mathbf{m A}$.

4. From Fig. S1, it looks like the ZnS NPs have a pretty wide size distribution. Some indication of what this is would be useful. 
According to this comment, the following sentences were added in the revised text.

From the Figure S1, it looks like the ZnS NPs have a pretty wide distribution. In addition, the spectrum exhibits a wavelength tail extending up to about 600 nm. A possible explanation for the broad PL spectrum is the presence of a wide size distribution of NPs in the sample.

\section{Minor revisions}

1. The writing is generally clear, but there are a number of minor stylistic and grammatical errors that could be fixed by a native English speaker. ("the" vs. "a", for example).

A number of minor stylistic and grammatical errors were revised and were fixed by a native English speaker.

2. P. 4, line 5. I don't understand the "2 counts.s-1" in connection with the reference electrode.

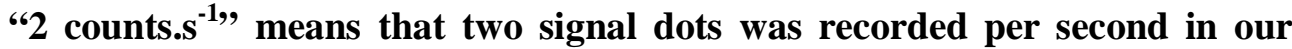
ECL instrument. This is a mistaking. Therefore, " 2 counts. ${ }^{-1}$ ", was deleted.

3. p. 5, line 5. l'd recommend "more efficient" rather than "highly efficient" in the absence of any quantum efficiency information.

The words "highly efficient" were revised as "more efficient".

\section{Reviewer \#66}

1. Water will be electrolyzed when scanned negatively to $-2.0 \mathrm{~V}$. The author did not mention how to avoid it during the experiments.

It is right. Water will be electrolyzed at a Pt electrode, even if in $0.10 \mathrm{M} \mathrm{NaOH}$, when a potential is scanned negatively from $-1.25 \mathrm{~V}$. It could not be avoided during our present experiments. If the carbon electrode including glassy carbon electrode and 
graphite electrode was used in higher concentration of $\mathrm{NaOH}$, the electrolyzation of the aqueous solvent will be lessened. Fortunately, the ECL emission was not greatly affected by the electrolyzation of the aqueous solvent, as described in other papers. Therefore, the following sentences were added in the revised text.

Furthermore, the ECL intensity at $+\mathbf{0 . 8 6} \mathrm{V}$ increased as the start scanning potential from $-1.25 \mathrm{~V}$ to $-\mathbf{2 . 0} \mathrm{V}$. This indicates that the more negative potential applied benefit this ECL process and the ECL process is directly related to the electrochemical reduce process. It was found that the water was electrolyzed in 0.10 M NaOH at the potential more negative than $-1.0 \mathrm{~V}$ (data not shown). ${ }^{17}$ Although water was electrochemically reduced in $0.10 \mathrm{M} \mathrm{NaOH}$ solution containing $1.0 \mathrm{mM} \mathrm{ZnS}$ NPs at the potential more negative than $-1.0 \mathrm{~V}$ (data not shown), a stable and strong ECL emission was observed. This may be caused by a adsorption of $\mathrm{ZnS}$ NPs on the electrode.

2. The author states that nanoparticles are core/shell structure and the passivation of $\mathrm{Zn}(\mathrm{OH})_{2}$ on the surface of $\mathrm{ZnS}$ NPs, however, data may be insufficient to confirm this conclusion. Some characterization such as XRD or HRTEM may be helpful.

The characterization of $\mathrm{ZnS}$ NPs in the absence and presence of $0.10 \mathrm{M} \mathrm{NaOH}$. using HRTEM, XRD and EDX were performed to confirm the core/shell structure and the passivation of $\mathrm{Zn}(\mathrm{OH})_{2}$ on the surface of $\mathrm{ZnS}$ NPs. The results and discussion were presented in revised text, here, not described duo to too long.

3. What is the concentration of the as-prepared ZnS NPs colloidal solution? Did the author study the relationship between the concentration of ZnS and the ECL intensity?

The concentration of the as-prepared $\mathrm{ZnS}$ colloidal solution was expressed as the 
concentration of chemical composition of $\mathrm{ZnS}$ NPs. When $\mathrm{ZnS}$ colloidal solution was prepared using the moler ratio of $\mathrm{Zn}^{2+}$ and $\mathrm{S}^{2-}$ of $1: 1$, it can be regarded that the $\mathrm{Zn}^{2+}$ and $\mathrm{S}^{2-}$ were totally transformed to $\mathrm{ZnS}$ NPs. So, The concentration of the as-prepared $\mathrm{ZnS}$ colloidal solution was expressed as the calculated concentration,which was $0.0010 \mathrm{M}$.Therefore, the following sentences were added in Experimental Section, the revised text.

The concentration of $\mathrm{ZnS}$ NPs colloidal solution was calculated from above procedure and regarded as $0.0010 \mathrm{M}$.

The relationship between the concentration of ZnS NPs and the ECL intensity was studied. The results and discussion were presented in revised text and Figure S4 presenting the relationship was added in the Supporting Information.

The dependence of the ECL intensity on the concentration of ZnS NPs in 0.10M NaOH containing $0.010 \mathrm{M} \mathrm{K}_{2} \mathrm{~S}_{2} \mathrm{O}_{8}$ was investigated. It was found that the ECL intensity linearly increased along with the concentration of ZnS NPs in the range from $1.2 \times 10^{-4}$ to $1.0 \times 10^{-3} \mathrm{M}$ ( as shown in Figure $\mathrm{S} 4$ in the Supporting Information). This suggests that $\mathrm{ZnS}$ NPs in $0.10 \mathrm{M}$ NaOH containing $0.010 \mathrm{M}$ K2S2O8 is a potential applications in analytical chemistry. It is noted that the efficiency of the NPs should be improved.

4. No scale on Y-axis in Figure.

The Y-axis was marked in Figure 1. 